\title{
GENERALIZED SKEW DERIVATIONS AS JORDAN HOMOMORPHISMS ON MULTILINEAR POLYNOMIALS
}

\author{
Vincenzo De Filippis
}

\begin{abstract}
Let $\mathcal{R}$ be a prime ring of characteristic different from 2, $\mathcal{Q}_{r}$ be its right Martindale quotient ring and $\mathcal{C}$ be its extended centroid. Suppose that $\mathcal{G}$ is a nonzero generalized skew derivation of $\mathcal{R}$, $\alpha$ is the associated automorphism of $\mathcal{G}, f\left(x_{1}, \ldots, x_{n}\right)$ is a non-central multilinear polynomial over $\mathcal{C}$ with $n$ non-commuting variables and $\mathcal{S}=$ $\left\{f\left(r_{1}, \ldots, r_{n}\right) \mid r_{1}, \ldots, r_{n} \in \mathcal{R}\right\}$. If $\mathcal{G}$ acts as a Jordan homomorphism on $\mathcal{S}$, then either $\mathcal{G}(x)=x$ for all $x \in \mathcal{R}$, or $\mathcal{G}=\alpha$
\end{abstract}

\section{Introduction}

In all that follows let $\mathcal{R}$ be a prime ring, $\mathcal{Z}(\mathcal{R})$ the center of $\mathcal{R}, \mathcal{Q}_{r}$ be the right Martindale quotient ring of $\mathcal{R}$ and $\mathcal{C}=\mathcal{Z}\left(\mathcal{Q}_{r}\right)$ be the center of $\mathcal{Q}_{r} . \mathcal{C}$ is usually called the extended centroid of $\mathcal{R}$ and is a field when $\mathcal{R}$ is a prime ring. It should be remarked that $\mathcal{Q}_{r}$ is a centrally closed prime $\mathcal{C}$-algebra. We refer the reader to $[6]$ for the definitions and the related properties of these objects.

We recall that an additive map $d$ on $\mathcal{R}$ is called a derivation if $d(x y)=$ $d(x) y+x d(y)$ for all $x, y \in \mathcal{R}$. Starting from this definition we may introduce another concept of an additive function which generalizes derivations: the additive map $\mathcal{G}$ of $\mathcal{R}$ is said to be a generalized derivation if $\mathcal{G}(x y)=\mathcal{G}(x) y+x d(y)$ for all $x, y \in \mathcal{R}$, where $d$ is a derivation of $\mathcal{R}$ (usually $\mathcal{G}$ is said to be a generalized derivation associated with $d$ ). Obviously, any derivation of $\mathcal{R}$ and any map of $\mathcal{R}$ with form $f(x)=a x+x b$ for some $a, b \in \mathcal{R}$, are both generalized derivations. The latter are usually called inner generalized derivations. We would like to point out that one of the leading roles in the development of the theory of generalized derivations is played by the inner generalized derivations.

We say that an additive map $\mathcal{F}$ acts as a homomorphism on a subset $\mathcal{T} \subseteq \mathcal{R}$, if $\mathcal{F}(x y)=\mathcal{F}(x) \mathcal{F}(y)$ for all $x, y \in \mathcal{T} ; \mathcal{F}$ acts as an anti-homomorphism on $\mathcal{T}$, if $\mathcal{F}(x y)=\mathcal{F}(y) \mathcal{F}(x)$ for all $x, y \in \mathcal{T}$; finally $\mathcal{F}$ acts as a Jordan homomorphism on $\mathcal{T}$ if $\mathcal{F}\left(x^{2}\right)=\mathcal{F}(x)^{2}$ for all $x \in \mathcal{T}$. Obviously any additive map, which is a homomorphism or an anti-homomorphism, is a Jordan homomorphism. On

Received May 19, 2014; Revised October 10, 2014

2010 Mathematics Subject Classification. 16W25, 16N60.

Key words and phrases. polynomial identity, generalized skew derivation, prime ring. 
the other hand, in [20, p. 50] Herstein proves that in case $\mathcal{R}$ is a prime ring of characteristic different from 2 , any Jordan homomorphism on $\mathcal{R}$ is either a homomorphism or an anti-homomorphism of $\mathcal{R}$.

In [7, Theorem 3] Bell and Kappe prove that if $d$ is a derivation of a prime ring $R$ which acts as a homomorphism or anti-homomorphism on a non-zero right ideal of $R$, then $d=0$ on $R$.

In [34] Wang and You extend this result to a Lie ideal $\mathcal{L}$ of a prime ring $\mathcal{R}$ with characteristic different from 2 . They prove that there is no non-zero derivation acting as a homomorphism or anti-homomorphism on $\mathcal{L}$, unless when $\mathcal{L} \subseteq \mathcal{Z}(\mathcal{R})$

Recently, Rehman (in [31]) and Albaş and Argaç (in [1]) study the case when the derivation $d$ is replaced by a generalized derivation $\mathcal{G}$ associated to a derivation $d$. In both papers it is proved that if $0 \neq \mathcal{G}$ acts as a homomorphism or anti-homomorphism on $\mathcal{I}$, a non-zero ideal of the prime ring $\mathcal{R}$, then either $d=0$ or $\mathcal{R}$ is commutative. In particular, if assume that $\mathcal{G}$ acts as a homomorphism on $\mathcal{I}$, then either $\mathcal{R}$ is commutative or $\mathcal{G}$ is the identity map on $\mathcal{R}$. On the other hand, if assume that $\mathcal{G}$ acts as an anti-homomorphism on $\mathcal{I}$, then $\mathcal{R}$ is commutative.

Following this line of investigation, in [17] we obtain the following result: Let $\mathcal{R}$ be a prime ring, $\mathcal{L}$ a non-central Lie ideal of $\mathcal{R}$ and $\mathcal{F}$ a non-zero generalized derivation of $\mathcal{R}$. If $\mathcal{F}$ acts as a Jordan homomorphism on $\mathcal{L}$, then either $\mathcal{F}(x)=x$ for all $x \in \mathcal{R}$, or $\operatorname{char}(R)=2, \mathcal{R}$ satisfies the standard identity $s_{4}\left(x_{1}, x_{2}, x_{3}, x_{4}\right), \mathcal{L}$ is commutative and $u^{2} \in \mathcal{Z}(\mathcal{R})$ for any $u \in \mathcal{L}$.

Generalized derivations and generalized $(\alpha, \beta)$-derivations as homomorphisms, anti-homomorphisms or Lie homomorphisms in prime rings, as well as derivations as homomorphisms or anti-homomorphisms in $\sigma$-prime rings, have also been discussed in $[2,3,4,5,30,32,36]$.

Our work is then motived by the previous results. In the current presentation we will continue the study of additive maps which act as Jordan homomorphisms. We will now recall the definition of generalized skew derivations of $\mathcal{R}$. Let $\mathcal{R}$ be an associative ring and $\alpha$ be an automorphism of $\mathcal{R}$. An additive map $d: \mathcal{R} \longrightarrow \mathcal{R}$ is called a skew derivation of $\mathcal{R}$ if

$$
d(x y)=d(x) y+\alpha(x) d(y)
$$

for all $x, y \in \mathcal{R}$ and $\alpha$ is called an associated automorphism of $d$. An additive map $\mathcal{G}: \mathcal{R} \longrightarrow \mathcal{R}$ is said to be a generalized skew derivation of $R$ if there exists a skew derivation $d$ of $\mathcal{R}$ with associated automorphism $\alpha$ such that

$$
\mathcal{G}(x y)=\mathcal{G}(x) y+\alpha(x) d(y)
$$

for all $x, y \in \mathcal{R}, d$ is said to be an associated skew derivation of $\mathcal{G}$ and $\alpha$ is called an associated automorphism of $\mathcal{G}$. The definition of generalized skew derivations is a unified notion of skew derivation and generalized derivation, which are considered as classical additive maps of non-associative algebras, 
have been investigated by many researchers from various views, see $[8,9,10]$, [11], [25], [27].

More precisely speaking, we will prove:

Theorem 1. Let $\mathcal{R}$ be a prime ring of characteristic different from $2, \mathcal{Q}_{r}$ be its right Martindale quotient ring and $\mathcal{C}$ be its extended centroid. Suppose that $\mathcal{G}$ is a nonzero generalized skew derivation of $\mathcal{R}, \alpha$ is the associated automorphism of $\mathcal{G}, f\left(x_{1}, \ldots, x_{n}\right)$ is a non-central multilinear polynomial over $\mathcal{C}$ with $n$ noncommuting variables and $\mathcal{S}=\left\{f\left(r_{1}, \ldots, r_{n}\right) \mid r_{1}, \ldots, r_{n} \in \mathcal{R}\right\}$. If $\mathcal{G}$ acts as a Jordan homomorphism on $\mathcal{S}$, then either $\mathcal{G}(x)=x$ for all $x \in \mathcal{R}$, or $\mathcal{G}=\alpha$.

It is well known that automorphisms, derivations and skew derivations of $\mathcal{R}$ can be extended both to $\mathcal{Q}_{r}$. Chang in [8] extends the definition of generalized skew derivation to the right Martindale quotient ring $\mathcal{Q}_{r}$ of $\mathcal{R}$ as follows: by a (right) generalized skew derivation we mean an additive map $\mathcal{G}: \mathcal{Q}_{r} \longrightarrow \mathcal{Q}_{r}$ such that $\mathcal{G}(x y)=\mathcal{G}(x) y+\alpha(x) d(y)$ for all $x, y \in \mathcal{Q}_{r}$, where $d$ is a skew derivation of $\mathcal{R}$ and $\alpha$ is an automorphism of $\mathcal{R}$. Moreover, there exists $\mathcal{G}(1)=$ $a \in \mathcal{Q}_{r}$ such that $\mathcal{G}(x)=a x+d(x)$ for all $x \in \mathcal{R}$. In other words, any generalized skew derivation $\mathcal{G}$ of $\mathcal{R}$ can be extended to $\mathcal{Q}_{r}$. We will adopt the following notation:

$$
f\left(x_{1}, \ldots, x_{n}\right)=x_{1} x_{2} \ldots x_{n}+\sum_{\sigma \in S_{n}, \sigma \neq i d} \alpha_{\sigma} x_{\sigma(1)} x_{\sigma(2)} \cdots x_{\sigma(n)}
$$

for some $\alpha_{\sigma} \in \mathcal{C}$ and

$$
\mathcal{S}=\left\{f\left(r_{1}, \ldots, r_{n}\right) \mid r_{1}, \ldots, r_{n} \in \mathcal{R}\right\} .
$$

We always suppose that $\mathcal{G} \neq 0, \operatorname{char}(\mathcal{R}) \neq 2$ and $f\left(x_{1}, \ldots, x_{n}\right)$ is non-central valued in $\mathcal{R}$.

We start with the following easy result:

Lemma 1.1. Let $\mathcal{R}$ be a prime ring of characteristic different from $2, \mathcal{G}$ a nonzero generalized skew derivation of $\mathcal{R}, \alpha$ the associated automorphism of $\mathcal{G}$. If $\mathcal{G}$ acts as a Jordan homomorphism on $\mathcal{R}$, then either $\mathcal{G}(x)=x$ for all $x \in \mathcal{R}$, or $\mathcal{G}=\alpha$.

Proof. Since $\operatorname{char}(\mathcal{R}) \neq 2$, then it is known that $\mathcal{G}$ is either a homomorphism or an anti-homomorphism of $\mathcal{R}$. In light of this we divide the proof into two cases:

Case 1. Assume firstly that $\mathcal{G}$ is a homomorphism of $\mathcal{R}$, that is

$$
\mathcal{G}(x y)=\mathcal{G}(x) \mathcal{G}(y), \quad \forall x, y \in \mathcal{R} .
$$

On the other hand we know that $\mathcal{G}(x y)=\mathcal{G}(x) y+\alpha(x) d(y)$. Comparing this last relation with (1.1) we obtain

$$
\mathcal{G}(x)(y-\mathcal{G}(y))+\alpha(x) d(y)=0, \quad \forall x, y \in \mathcal{R} .
$$


Recall that there exists $a \in \mathcal{Q}_{r}$ such that $\mathcal{G}(x)=a x+d(x)$ for all $x \in \mathcal{R}$. Hence by (1.2) we get

$$
\mathcal{G}(x)(y-a y-d(y))+\alpha(x) d(y)=0, \quad \forall x, y \in \mathcal{R}
$$

that is

$$
(\alpha(x)-\mathcal{G}(x)) d(y)+\mathcal{G}(x)(y-a y)=0, \quad \forall x, y \in \mathcal{R}
$$

Replacing $y$ by $y r$ in (1.3), for any $r \in R$, and using again the relation (1.3), it follows

$$
(\alpha(x)-\mathcal{G}(x)) \alpha(y) d(r)=0, \quad \forall x, y, r \in \mathcal{R} .
$$

Therefore, by the primeness of $\mathcal{R}$, one has that either $\mathcal{G}=\alpha$ or $d=0$. In this last case, $\mathcal{G}(x)=a x$ is a generalized derivation of $\mathcal{R}$. Hence, by using the result contained in Proposition 1 of [17] and since $\mathcal{G} \neq 0$, we conclude that $\mathcal{G}(x)=x$, for all $x \in \mathcal{R}$.

Case 2. Let now $\mathcal{G}$ be an anti-homomorphism of $\mathcal{R}$, that is

$$
\mathcal{G}(x y)=\mathcal{G}(y) \mathcal{G}(x), \quad \forall x, y \in \mathcal{R} .
$$

Moreover we may assume $d \neq 0$, otherwise we may conclude as above that $\mathcal{G}(x)=x$ for all $x \in \mathcal{R}$.

Comparing (1.4) with $\mathcal{G}(x y)=\mathcal{G}(x) y+\alpha(x) d(y)$, we get

$$
\mathcal{G}(x) y+\alpha(x) d(y)=\mathcal{G}(y) \mathcal{G}(x) .
$$

Replacing $x$ with $x y$ in (1.5) and using (1.4) it follows

$$
\alpha(x y) d(y)=\mathcal{G}(y) \alpha(x) d(y), \forall x, y \in \mathcal{R} .
$$

Now replace $x$ by $r x$ in (1.6), for any $r \in R$, thus the following holds

$$
\alpha(r x y) d(y)=\mathcal{G}(y) \alpha(r x) d(y), \forall x, y, r \in \mathcal{R}
$$

and by using (1.6) in (1.7) we also have

$$
\alpha(r) \mathcal{G}(y) \alpha(x) d(y)=\mathcal{G}(y) \alpha(r) \alpha(x) d(y), \forall x, y, r \in \mathcal{R}
$$

that is $[\mathcal{G}(y), \alpha(\mathcal{R})] \alpha(\mathcal{R}) d(y)=(0)$, for all $y \in \mathcal{R}$. Since $\mathcal{R}$ is prime, it follows that, for any $y \in \mathcal{R}$, either $d(y)=0$ or $\mathcal{G}(y) \in \mathcal{Z}(\mathcal{R})$.

Assume there exists an element $u \in \mathcal{R}$ such that $\mathcal{G}(u) \in \mathcal{Z}(\mathcal{R})$. Therefore, for any $x \in \mathcal{R}$, by our assumption, we have

$$
\mathcal{G}(u x)=\mathcal{G}(x) \mathcal{G}(u)=\mathcal{G}(u) \mathcal{G}(x)
$$

and replacing $x$ by $x r$ in (1.8)

$$
\mathcal{G}(u x) r+\alpha(u x) d(r)=\mathcal{G}(u) \mathcal{G}(x) r+\mathcal{G}(u) \alpha(x) d(r)
$$

that is

$$
\mathcal{G}(x) \mathcal{G}(u) r+\alpha(u x) d(r)=\mathcal{G}(u) \mathcal{G}(x) r+\mathcal{G}(u) \alpha(x) d(r)
$$


and since $\mathcal{G}(u) \in \mathcal{Z}(\mathcal{R})$, one has

$$
\alpha(u) \alpha(x) d(r)=\mathcal{G}(u) \alpha(x) d(r), \forall x, r \in \mathcal{R}
$$

which means

$$
(\mathcal{G}(u)-\alpha(u)) \alpha(\mathcal{R}) d(\mathcal{R})=(0) .
$$

Since $d \neq 0$ and by the primeness of $\mathcal{R}$, it follows that $\alpha(u)=\mathcal{G}(u) \in \mathcal{Z}(\mathcal{R})$, which implies $u \in \mathcal{Z}(\mathcal{R})$.

The previous argument means that for any $y \in \mathcal{R}$, either $d(y)=0$ or $y \in$ $\mathcal{Z}(\mathcal{R})$. In any case we have that $[d(y), y]=0$ for all $y \in \mathcal{R}$. In this case, by [28, Theorem 2] and since $d \neq 0, \mathcal{R}$ must be commutative, so that $\mathcal{G}$ is a homomorphism of $\mathcal{R}$ and by Case 1 we get $\mathcal{G}=\alpha$.

\section{The case of inner generalized skew derivations}

In this section we will deal with the case when $\mathcal{G}$ is an inner generalized skew derivation induced by the elements $a, b \in \mathcal{R}$ and $\alpha \in \operatorname{Aut}(\mathcal{R})$, that is $\mathcal{G}(x)=a x+\alpha(x) b$ for all $x \in \mathcal{R}$. In this sense, our aim is to prove the following:

Proposition 2.1. Let $\mathcal{R}$ be a prime ring of characteristic different from 2 and $f\left(x_{1}, \ldots, x_{n}\right)$ be a non-central multilinear polynomial over $\mathcal{C}$ with $n$ noncommuting variables and $\mathcal{S}=\left\{f\left(r_{1}, \ldots, r_{n}\right) \mid r_{1}, \ldots, r_{n} \in \mathcal{R}\right\}$. Let $a, b \in \mathcal{R}$ and $\alpha \in \operatorname{Aut}(\mathcal{R})$ such that $\mathcal{G}(x)=a x+\alpha(x) b$ for all $x \in \mathcal{R}$. If $\mathcal{G}\left(x^{2}\right)=\mathcal{G}(x)^{2}$ for all $x \in S$, then either $\mathcal{G}(x)=x$ for all $x \in \mathcal{R}$, or $\mathcal{G}=\alpha$.

\subsection{The matrix case}

Let us first consider the case when $\mathcal{R}=\mathcal{M}_{m}(\mathcal{K})$ is the algebra of $m \times m$ matrices over a field $\mathcal{K}$ of characteristic different from 2 . Note that the set $f(\mathcal{R})=\left\{f\left(r_{1}, \ldots, r_{n}\right) \mid r_{1}, \ldots, r_{n} \in \mathcal{R}\right\}$ is invariant under the action of all inner automorphisms of $\mathcal{R}$. Let us write $r=\left(r_{1}, \ldots, r_{n}\right) \in \mathcal{R} \times \mathcal{R} \times \cdots \times$ $\mathcal{R}=\mathcal{R}^{n}$. Then for any inner automorphism $\varphi$ of $\mathcal{M}_{m}(\mathcal{K})$, we get that $\underline{r}=$ $\left(\varphi\left(r_{1}\right), \ldots, \varphi\left(r_{n}\right)\right) \in \mathcal{R}^{n}$ and $\varphi(f(r))=f(\underline{r}) \in f(\mathcal{R})$. As usual, we denote the matrix unit having 1 in $(i, j)$-entry and zero elsewhere by $e_{i j}$.

Let us recall some results from [24] and [26]. Let $\mathcal{T}$ be a ring with 1 and let $e_{i j} \in \mathcal{M}_{m}(\mathcal{T})$ be the matrix unit having 1 in $(i, j)$-entry and zero elsewhere. For a sequence $u=\left(\mathcal{A}_{1}, \ldots, \mathcal{A}_{n}\right)$ in $\mathcal{M}_{m}(\mathcal{T})$, the value of $u$ is defined to be the product $|u|=\mathcal{A}_{1} \mathcal{A}_{2} \cdots \mathcal{A}_{n}$ and $u$ is nonvanishing if $|u| \neq 0$. For a permutation $\sigma$ of $\{1,2, \ldots, n\}$, we write $u^{\sigma}=\left(\mathcal{A}_{\sigma(1)}, \ldots, \mathcal{A}_{\sigma(n)}\right)$. We call $u$ simple if it is of the form $u=\left(a_{1} e_{i_{1} j_{1}}, \ldots, a_{n} e_{i_{n} j_{n}}\right)$, where $a_{i} \in \mathcal{T}$. A simple sequence $u$ is called even if for some $\sigma,\left|u^{\sigma}\right|=b e_{i i} \neq 0$, and odd if for some $\sigma,\left|u^{\sigma}\right|=b e_{i j} \neq 0$, where $i \neq j$. We have:

Fact 2.2 ([24, Lemma $])$. Let $\mathcal{T}$ be a $\mathcal{K}$-algebra with 1 and let $\mathcal{R}=\mathcal{M}_{m}(\mathcal{T})$, $m \geq 2$. Suppose that $g\left(x_{1}, \ldots, x_{n}\right)$ is a multilinear polynomial over $\mathcal{K}$ such 
that $g(u)=0$ for all odd simple sequences $u$. Then $g\left(x_{1}, \ldots, x_{n}\right)$ is centrally valued on $\mathcal{R}$.

Fact $2.3([26$, Lemma 2$])$. Let $\mathcal{T}$ be a $\mathcal{K}$-algebra with 1 and let $\mathcal{R}=\mathcal{M}_{m}(\mathcal{T})$, $m \geq 2$. Suppose that $g\left(x_{1}, \ldots, x_{n}\right)$ is a multilinear polynomial over $\mathcal{K}$. Let $u=\left(\mathcal{A}_{1}, \ldots, \mathcal{A}_{n}\right)$ be a simple sequence from $\mathcal{R}$.

(a) If $u$ is even, then $g(u)$ is a diagonal matrix.

(b) If $u$ is odd, then $g(u)=a e_{p q}$ for some $a \in \mathcal{T}$ and $p \neq q$.

Fact 2.4. Since $f\left(x_{1}, \ldots, x_{n}\right)$ is not centrally valued on $\mathcal{R}$, then by Fact 2.2 there exists an odd simple sequence $r=\left(r_{1}, \ldots, r_{n}\right)$ from $\mathcal{R}$ such that $f(r)=$ $f\left(r_{1}, \ldots, r_{n}\right) \neq 0$. By Fact $2.3, f(r)=\beta e_{p q}$, where $0 \neq \beta \in \mathcal{C}$ and $p \neq q$. Since $f\left(x_{1}, \ldots, x_{n}\right)$ is a multilinear polynomial and $\mathcal{C}$ is a field, we may assume that $\beta=1$. Now, for distinct $i, j$, let $\sigma \in S_{n}$ be such that $\sigma(p)=i$ and $\sigma(q)=j$, and let $\psi$ be the automorphism of $\mathcal{R}$ defined by $\psi\left(\sum_{s, t} \xi_{s t} e_{s t}\right)=\sum_{s, t} \xi_{s t} e_{\sigma(s) \sigma(t)}$. Then $f(\psi(r))=f\left(\psi\left(r_{1}\right), \ldots, \psi\left(r_{n}\right)\right)=\psi(f(r))=\beta e_{i j}=e_{i j}$.

We start with the following (Lemma 1.5 in [18]):

Lemma 2.5. Let $\mathcal{H}$ be an infinite field, $m$ be a positive integer with $m \geq 2$ and $\mathcal{M}_{m}(\mathcal{H})$ be the algebra of $m \times m$ matrices over $\mathcal{H}$. If $\mathcal{A}_{1}, \ldots, \mathcal{A}_{k}$ are not scalar matrices in $\mathcal{M}_{m}(\mathcal{H})$, then there exists some invertible matrix $\mathcal{B} \in \mathcal{M}_{m}(\mathcal{H})$ such that each matrix $\mathcal{B A}_{1} \mathcal{B}^{-1}, \ldots, \mathcal{B A}_{k} \mathcal{B}^{-1}$ has all non-zero entries.

Lemma 2.6. Let $\mathcal{H}$ be an infinite field, $m$ be a positive integer with $m \geq 2$ and $\mathcal{R}=\mathcal{M}_{m}(\mathcal{H})$ be the algebra of $m \times m$ matrices over $\mathcal{H}$. If there exists $a \in \mathcal{R}$ such that $a u^{2}=(a u)^{2}$ for all $u \in \mathcal{S}$, then either $a=0$ or $a=I$, the identity matrix in $\mathcal{R}$.

Proof. If $a \in \mathcal{Z}(\mathcal{R})$ then $\left(a-a^{2}\right) u^{2}=0$ follows by our assumption. Since $\left(a-a^{2}\right) \in \mathcal{Z}(\mathcal{R})$, we have that either $a-a^{2}=0$ or $f\left(x_{1}, \ldots, x_{n}\right)^{2}$ is a polynomial identity for $\mathcal{R}$.

In the first case either $a=0$ or $a=I$, the identity matrix in $\mathcal{R}$. In the latter one, by Main Theorem' in [15] it follows that $f\left(x_{1}, \ldots, x_{n}\right)$ is a polynomial identity for $R$, which is a contradiction.

Thus we may assume that $a$ is not a scalar matrix and proceed to have a contradiction. By Lemma 2.5, there exists some invertible matrix $\mathcal{B} \in M_{m}(\mathcal{H})$ such that $\mathcal{B} \mathcal{B}^{-1}$, has all nonzero entries. Denote by $\varphi(x)=\mathcal{B} x \mathcal{B}^{-1}$ the inner automorphism induced by $\mathcal{B}$. Since $f(\mathcal{R})$ is invariant under the action of all inner automorphisms of $\mathcal{R}$, then $\varphi(a) u^{2}=(\varphi(a) u)^{2}$ for all $u \in \mathcal{S}$. Let us write $\varphi(a)=\sum_{h l} a_{h l} e_{h l}$ for $0 \neq a_{h l} \in \mathcal{H}$. Since $e_{i j} \in \mathcal{S}$ for all $i \neq j$, then, for any $i \neq j$, we have $0=\left(\varphi(a) e_{i j}\right)^{2}$. In particular, the $(j, i)$-entry of $\varphi(a)$ is zero, which is a contradiction.

Claim 2.7. We remark that, analogously one can prove that if $u^{2} a=(u a)^{2}$ for all $u \in \mathcal{S}$, then either $a=0$ or $a=I$. 
Lemma 2.8. Let $\mathcal{H}$ be an infinite field, $m$ be a positive integer with $m \geq 2$ and $\mathcal{R}=\mathcal{M}_{m}(\mathcal{H})$ be the algebra of $m \times m$ matrices over $\mathcal{H}$. If there exist $a, b \in \mathcal{R}$ such that $a u^{2}+u^{2} b=(a u+u b)^{2}$ for all $u \in \mathcal{S}$, then $a, b \in \mathcal{Z}(\mathcal{R})$ with $a+b=I$, the identity matrix in $\mathcal{R}$.

Proof. If either $a \in \mathcal{Z}(\mathcal{R})$ or $b \in \mathcal{Z}(\mathcal{R})$, then the conclusion follows from Lemma 2.6 and Claim 2.7. Thus we may assume that both $a$ and $b$ are not scalar matrices and proceed to have a contradiction. By Lemma 2.5, there exists some invertible matrix $\mathcal{B} \in M_{m}(\mathcal{H})$ such that each matrix $\mathcal{B a B}^{-1}, \mathcal{B b}^{-1}$ has all nonzero entries. Denote by $\varphi(x)=\mathcal{B} x \mathcal{B}^{-1}$ the inner automorphism induced by $\mathcal{B}$. As above we notice that $\varphi(a) u^{2}+u^{2} \varphi(b)=(\varphi(a) u+u \varphi(b))^{2}$, for all $u \in \mathcal{S}$. Moreover we write $\varphi(a)=\sum_{h l} a_{h l} e_{h l}$ and $\varphi(b)=\sum_{h l} b_{h l} e_{h l}$ for $0 \neq a_{h l}, 0 \neq b_{h l} \in \mathcal{H}$. Since $e_{i j} \in f(\mathcal{R})$ for all $i \neq j$, then for any $i \neq j$

$$
0=\left(\varphi(a) e_{i j}+e_{i j} \varphi(b)\right)^{2} .
$$

In particular, it follows simultaneously that the $(j, i)$-entry of $a$ is zero and $(j, i)$-entry of $b$ is zero, which is a contradiction.

Lemma 2.9. Let $\mathcal{H}$ be an infinite field, $m$ be a positive integer with $m \geq 2$ and $\mathcal{R}=\mathcal{M}_{m}(\mathcal{H})$ be the algebra of $m \times m$ matrices over $\mathcal{H}$. Assume there exist $0 \neq a, b, q \in \mathcal{R}$ such that $q$ is an invertible matrix such that $\mathcal{G}(x)=a x+q x q^{-1} b$ for all $x \in \mathcal{R}$. If $q^{-1} a \in \mathcal{Z}(\mathcal{R})$ and $\mathcal{G}\left(x^{2}\right)=\mathcal{G}(x)^{2}$ for all $x \in \mathcal{S}$, then either $\mathcal{G}(x)=x$ for all $x \in \mathcal{R}$ or $\mathcal{G}(x)=q x q^{-1}$ for all $x \in \mathcal{R}$.

Proof. We notice that in case $q^{-1} b \in \mathcal{Z}(\mathcal{R})$ then the conclusion follows from Lemma 2.6. Therefore we assume $q^{-1} b$ is not a scalar matrix.

We divide the proof into two cases.

Suppose first that $a+b=\lambda \in \mathcal{Z}(\mathcal{R})$. Thus, since $q^{-1} a \in \mathcal{Z}(\mathcal{R})$ and by easy computations, we note that $\mathcal{G}(x)=\lambda q x q^{-1}$ and $\lambda \neq 0$ since $\mathcal{G} \neq 0$. By our assumption it follows $\lambda u^{2}=\lambda^{2} u^{2}$ for all $u \in \mathcal{S}$. Since $\lambda \in \mathcal{Z}(\mathcal{R})$ and $f\left(x_{1}, \ldots, x_{n}\right)$ is not a polynomial identity for $\mathcal{R}$, by the same argument in Lemma 2.6, we have that $\lambda=1$.

Assume now that $a+b$ is not a central matrix in $\mathcal{R}$, that is $q^{-1}(a+b) q \notin$ $\mathcal{Z}(\mathcal{R})$. Again by Lemma 2.5 , there exists some invertible matrix $\mathcal{B} \in M_{m}(\mathcal{H})$ such that each matrix $\mathcal{B}\left(q^{-1} b\right) \mathcal{B}^{-1}, \mathcal{B}\left(q^{-1}(a+b) q\right) \mathcal{B}^{-1}$ has all nonzero entries. Denote by $\varphi(x)=\mathcal{B} x \mathcal{B}^{-1}$ the inner automorphism induced by $\mathcal{B}$. Simulating the above argument we will write $\varphi\left(q^{-1} b\right)=\sum_{h l} q_{h l} e_{h l}, \varphi\left(q^{-1}(a+b) q\right)=$ $\sum_{h l} p_{h l} e_{h l}$ for $0 \neq q_{h l}, 0 \neq p_{h l} \in \mathcal{H}$. Moreover, for $e_{i j} \in f(\mathcal{R})$,

$$
0=\left(\varphi(a) e_{i j}+\varphi(q) e_{i j} \varphi\left(q^{-1} \varphi(b)\right)^{2} .\right.
$$

Both left multiplying by $\varphi\left(q^{-1}\right)$ and right multiplying by $e_{i j}$ the relation (2.1), we get

$$
0=\left(\varphi\left(q^{-1}\right) \varphi(a) e_{i j}+e_{i j} \varphi\left(q^{-1}\right) \varphi(b)\right) \varphi(q) e_{i j} \varphi\left(q^{-1}\right) \varphi(b) e_{i j}
$$

and since $q^{-1} a \in \mathcal{Z}(\mathcal{R})$, it follows that

$$
0=e_{i j} \varphi\left(q^{-1}\right)(\varphi(a)+\varphi(b)) \varphi(q) e_{i j} \varphi\left(q^{-1}\right) \varphi(b) e_{i j}
$$


In particular, either the $(j, i)$-entry of $\varphi\left(q^{-1}(a+b) q\right)$ is zero, or the $(j, i)$-entry of $\varphi\left(q^{-1} b\right)$ is zero, which is any case a contradiction.

Lemma 2.10. Let $\mathcal{H}$ be an infinite field, $m$ be a positive integer with $m \geq 2$ and $\mathcal{R}=\mathcal{M}_{m}(\mathcal{H})$ be the algebra of $m \times m$ matrices over $\mathcal{H}$. Assume there exist $0 \neq a, b, q \in \mathcal{R}$ such that $q$ is an invertible matrix such that $\mathcal{G}(x)=a x+q x q^{-1} b$ for all $x \in \mathcal{R}$. If $\mathcal{G}\left(x^{2}\right)=\mathcal{G}(x)^{2}$ for all $x \in \mathcal{S}$, then either $\mathcal{G}(x)=x$ for all $x \in \mathcal{R}$ or $\mathcal{G}(x)=q x q^{-1}$ for all $x \in \mathcal{R}$.

Proof. In light of Lemmas 2.6, 2.8 and 2.9 we may assume now that $q, q^{-1} a$ and $q^{-1} b$ are not scalar matrices. As above, by Lemma 2.5 , there exists some invertible matrix $\mathcal{B} \in M_{m}(\mathcal{H})$ such that each matrix $\mathcal{B} q \mathcal{B}^{-1}, \mathcal{B}\left(q^{-1} a\right) \mathcal{B}^{-1}$, $\mathcal{B}\left(q^{-1} b\right) \mathcal{B}^{-1}$ has all nonzero entries. In order to prove our result, without loss of generality we may suppose that each matrix $q, q^{-1} a$ and $q^{-1} b$ has all nonzero entries. Write $q=\sum_{h l} q_{h l} e_{h l}, q^{-1} a=\sum_{h l} p_{h l} e_{h l}$ and $q^{-1} b=\sum_{h l} v_{h l} e_{h l}$, for $0 \neq q_{h l}, 0 \neq p_{h l}, 0 \neq v_{h l} \in \mathcal{H}$. Moreover, for $e_{i j} \in f(\mathcal{R})$,

$$
0=\left(a e_{i j}+q e_{i j} q^{-1} b\right)^{2} \text {. }
$$

Left multiplying by $e_{i j} q^{-1}$ and right multiplying by $e_{i j}$ the relation $(2.2)$, we get

$$
0=e_{i j} q^{-1} a e_{i j} q e_{i j} q^{-1} b e_{i j}
$$

which leads to the contradiction $q_{j i} p_{j i} v_{j i}=0$.

Lemma 2.11. Let $\mathcal{K}$ be a field of characteristic different $2, m$ be a positive integer with $m \geq 2$ and $\mathcal{R}=\mathcal{M}_{m}(\mathcal{K})$ be the algebra of $m \times m$ matrices over $\mathcal{K}$. If there exist $0 \neq a, b, q \in \mathcal{R}$ such that $q$ is an invertible matrix such that $\mathcal{G}(x)=a x+q x q^{-1} b$ for all $x \in \mathcal{R}$. If $\mathcal{G}\left(x^{2}\right)=\mathcal{G}(x)^{2}$ for all $x \in \mathcal{S}$, then either $\mathcal{G}(x)=x$ for all $x \in \mathcal{R}$ or $\mathcal{G}(x)=q x q^{-1}$ for all $x \in \mathcal{R}$.

Proof. If one assumes that $\mathcal{K}$ is infinite, the conclusion follows from Lemma 2.10 .

Now let $\mathcal{H}$ be an infinite field which is an extension of the field $\mathcal{K}$ and let $\overline{\mathcal{R}}=\mathcal{M}_{m}(\mathcal{H}) \cong \mathcal{R} \otimes \mathcal{K} \mathcal{H}$. Note that the multilinear polynomial $f\left(x_{1}, \ldots, x_{n}\right)$ is central-valued on $\mathcal{R}$ if and only if it is central-valued on $\overline{\mathcal{R}}$. We observe that the generalized polynomial

$$
\begin{aligned}
\Phi\left(x_{1}, \ldots, x_{n}\right)= & a f\left(x_{1}, \ldots, x_{n}\right)^{2}+q f\left(x_{1}, \ldots, x_{n}\right)^{2} q^{-1} b \\
& -\left(a f\left(x_{1}, \ldots, x_{n}\right)+q f\left(x_{1}, \ldots, x_{n}\right) q^{-1} b\right)^{2}
\end{aligned}
$$

is a generalized polynomial identity for $\mathcal{R}$. Moreover, $\Phi\left(x_{1}, \ldots, x_{n}\right)$ is multihomogeneous of multi-degree $(2, \ldots, 2)$ in the indeterminates $x_{1}, \ldots, x_{n}$. On the other hand, the complete linearization of $\Phi\left(x_{1}, \ldots, x_{n}\right)$ leads to a multilinear generalized polynomial $\Theta\left(x_{1}, \ldots, x_{n}, y_{1}, \ldots, y_{n}\right)$, which is of the form

$$
\Theta\left(x_{1}, \ldots, x_{n}, x_{1}, \ldots, x_{n}\right)=2^{n} \Phi\left(x_{1}, \ldots, x_{n}\right) .
$$


Clearly, the multilinear polynomial $\Theta\left(x_{1}, \ldots, x_{n}, y_{1}, \ldots, y_{n}\right)$ is a generalized polynomial identity for $\mathcal{R}$ and $\overline{\mathcal{R}}$ too. Since $\operatorname{char}(\mathcal{K}) \neq 2$, we obtain $\Phi\left(r_{1}, \ldots\right.$, $\left.r_{n}\right)=0$ for all $r_{1}, \ldots, r_{n} \in \overline{\mathcal{R}}$, and the conclusion follows from Lemma 2.10 .

Lemma 2.12. Let $\mathcal{K}$ be a field of characteristic different $2, m$ be a positive integer with $m \geq 2$ and $\mathcal{R}=\mathcal{M}_{m}(\mathcal{K})$ be the algebra of $m \times m$ matrices over $\mathcal{K}$. If there exist $0 \neq a, b \in \mathcal{R}$ such that $\mathcal{G}(x)=a x+x b$ for all $x \in \mathcal{R}$. If $\mathcal{G}\left(x^{2}\right)=\mathcal{G}(x)^{2}$ for all $x \in \mathcal{S}$, then $\mathcal{G}(x)=x$ for all $x \in \mathcal{R}$. In particular one of the following cases occurs: either $a, b \in \mathcal{Z}(\mathcal{R})$ and $a+b=1$ or $a=0$ and $b=1$, or $a=1$ and $b=0$.

Proof. If one assumes that $\mathcal{K}$ is infinite, the conclusion follows from Lemma 2.8 .

Now let $\mathcal{H}$ be an infinite field which is an extension of the field $\mathcal{K}$ and let $\overline{\mathcal{R}}=\mathcal{M}_{m}(\mathcal{H}) \cong \mathcal{R} \otimes_{\mathcal{K}} \mathcal{H}$. Note that the multilinear polynomial $f\left(x_{1}, \ldots, x_{n}\right)$ is central-valued on $\mathcal{R}$ if and only if it is central-valued on $\overline{\mathcal{R}}$. We observe that the generalized polynomial

$$
\begin{aligned}
\Phi\left(x_{1}, \ldots, x_{n}\right)= & a f\left(x_{1}, \ldots, x_{n}\right)^{2}+f\left(x_{1}, \ldots, x_{n}\right)^{2} b \\
& -\left(a f\left(x_{1}, \ldots, x_{n}\right)+f\left(x_{1}, \ldots, x_{n}\right) b\right)^{2}
\end{aligned}
$$

is a generalized polynomial identity for $\mathcal{R}$. Moreover, $\Phi\left(x_{1}, \ldots, x_{n}\right)$ is multihomogeneous of multi-degree $(2, \ldots, 2)$ in the indeterminates $x_{1}, \ldots, x_{n}$. On the other hand, the complete linearization of $\Phi\left(x_{1}, \ldots, x_{n}\right)$ leads to a multilinear generalized polynomial $\Theta\left(x_{1}, \ldots, x_{n}, y_{1}, \ldots, y_{n}\right)$, which is of the form

$$
\Theta\left(x_{1}, \ldots, x_{n}, x_{1}, \ldots, x_{n}\right)=2^{n} \Phi\left(x_{1}, \ldots, x_{n}\right) .
$$

Clearly, the multilinear polynomial $\Theta\left(x_{1}, \ldots, x_{n}, y_{1}, \ldots, y_{n}\right)$ is a generalized polynomial identity for $\mathcal{R}$ and $\overline{\mathcal{R}}$ too. Since $\operatorname{char}(\mathcal{K}) \neq 2$, we obtain $\Phi\left(r_{1}, \ldots\right.$, $\left.r_{n}\right)=0$ for all $r_{1}, \ldots, r_{n} \in \overline{\mathcal{R}}$, and the conclusion follows from Lemma 2.8 .

We also premit the following useful Lemma, which is a reduction of Proposition in [19]:

Lemma 2.13. Let $\mathcal{R}$ be a prime ring of characteristic different $2, a \in \mathcal{R}$, $f\left(x_{1}, \ldots, x_{n}\right)$ a multilinear polynomial over $\mathcal{C}$, which is not central valued on $\mathcal{R}$. If af $\left(r_{1}, \ldots, r_{n}\right)+f\left(r_{1}, \ldots, r_{n}\right) a=0$ for all $r_{1}, \ldots, r_{n} \in \mathcal{R}$, then $a=0$.

\subsection{The proof of Proposition 2.1 in case of inner generalized deriva- tions}

The first part of the proof of Proposition 2.1 is devoted to the case when $\alpha$ is the identity map on $\mathcal{R}$, that is, there exist $a, b \in \mathcal{Q}_{r}$ such that $\mathcal{G}(x)=a x+x b$ for all $x \in \mathcal{R}$. It is not difficult to see that, if either $a \notin \mathcal{C}$ or $b \notin \mathcal{C}$, then the 
generalized polynomial

$$
\begin{aligned}
\Phi\left(x_{1}, \ldots, x_{n}\right)= & a f\left(x_{1}, \ldots, x_{n}\right)^{2}+f\left(x_{1}, \ldots, x_{n}\right)^{2} b \\
& -\left(a f\left(x_{1}, \ldots, x_{n}\right)+f\left(x_{1}, \ldots, x_{n}\right) b\right)^{2}
\end{aligned}
$$

is a non-trivial generalized polynomial identity for $\mathcal{R}$.

It follows from [12] that $\Phi\left(x_{1}, \ldots, x_{n}\right)$ is a non-trivial generalized polynomial identity for $\mathcal{Q}_{r}$. By the well-known Martindale's theorem of [29], $\mathcal{Q}_{r}$ is a primitive ring having nonzero socle with the field $\mathcal{C}$ as its associated division ring. By $[21$, p. 75$] \mathcal{Q}_{r}$ is isomorphic to a dense subring of the ring of linear transformations of a vector space $\mathcal{V}$ over $\mathcal{C}$, containing nonzero linear transformations of finite rank.

If $\operatorname{dim}_{\mathcal{C}} \mathcal{V}=k \geq 2$ is a finite positive integer, then $\mathcal{Q}_{r} \cong \mathcal{M}_{k}(\mathcal{C})$ and the conclusion follows from Lemma 2.12.

Assume now that $\operatorname{dim}_{\mathcal{C}} \mathcal{V}=\infty$. As in lemma 2 in [35], the set $f(\mathcal{R})=$ $\left\{f\left(r_{1}, \ldots, r_{n}\right) \mid r_{i} \in \mathcal{R}\right\}$ is dense on $\mathcal{R}$. By the fact that $\Phi\left(r_{1}, \ldots, r_{n}\right)=0$ is a generalized polynomial identity of $\mathcal{R}$, we know that $\mathcal{R}$ satisfies the generalized polynomial identity

$$
a x^{2}+x^{2} b-(a x+x b)^{2}
$$

and the required conclusion follows from [17].

We finally consider the case $a, b \in \mathcal{C}$, that is $\mathcal{G}(x)=\lambda x$ for all $x \in \mathcal{R}$, with $\lambda=a+b \in \mathcal{C}$. Then by $(2.5)$ one has that $\left(\lambda-\lambda^{2}\right) f\left(x_{1}, \ldots, x_{n}\right)^{2}$ is an identity for $\mathcal{R}$. Since $f\left(x_{1}, \ldots, x_{n}\right)$ is not an identity for $\mathcal{R}$, it follows that either $\lambda=0$ or $\lambda=1$. Moreover $\mathcal{G} \neq 0$ implies $\lambda=1$, that is $\mathcal{G}(x)=x$ for all $x \in \mathcal{R}$.

\subsection{The proof of Proposition 2.1 in case of inner associated auto- morphism}

Assume now that $\alpha$ is an $X$-inner automorphism of $\mathcal{R}$, that is, there exists an element $q \in \mathcal{Q}_{r}$ such that $\alpha(x)=q x q^{-1}$ for all $x \in \mathcal{R}$. In case $q \in \mathcal{C}$, the conclusion follows from Subsection 2.2. Thus we consider $q \notin \mathcal{C}$.

Hence $\mathcal{R}$ satisfies the generalized polynomial identity

$$
\begin{aligned}
\Phi\left(x_{1}, \ldots, x_{n}\right)= & a f\left(x_{1}, \ldots, x_{n}\right)^{2}+q f\left(x_{1}, \ldots, x_{n}\right)^{2} q^{-1} b \\
& -\left(a f\left(x_{1}, \ldots, x_{n}\right)+q f\left(x_{1}, \ldots, x_{n}\right) q^{-1} b\right)^{2} .
\end{aligned}
$$

If $q^{-1} b \in \mathcal{C}$, then $(2.7)$ reduces to

$$
(a+b) f\left(x_{1}, \ldots, x_{n}\right)^{2}=\left((a+b) f\left(x_{1}, \ldots, x_{n}\right)\right)^{2} .
$$

Moreover in case $a+b=\lambda \in \mathcal{C}$, then by (2.8) one has that $\left(\lambda-\lambda^{2}\right) f\left(x_{1}, \ldots, x_{n}\right)^{2}$ is an identity for $\mathcal{R}$. Since $f\left(x_{1}, \ldots, x_{n}\right)$ is not an identity for $\mathcal{R}$, it follows that either $\lambda=0$ or $\lambda=1$. Since $\mathcal{G} \neq 0$, we get $\lambda=1$ and $\mathcal{G}(x)=x$ for all $x \in \mathcal{R}$. 
Consider now the case $a+b \notin \mathcal{C}$. It follows that (2.8) is a non-trivial generalized polynomial identity for $\mathcal{R}$.

On the other hand, in case $\left\{1, q^{-1} b\right\}$ are linearly $\mathcal{C}$-independent, then $(2.7)$ is a non-trivial generalized polynomial identity for $\mathcal{R}$.

Therefore in any case $\Phi\left(x_{1}, \ldots, x_{n}\right)$ is a non-trivial generalized polynomial identity for $\mathcal{R}$. It follows from [12] that $\Phi\left(x_{1}, \ldots, x_{n}\right)$ is a non-trivial generalized polynomial identity for $\mathcal{Q}_{r}$. As above, by [29], $\mathcal{Q}_{r}$ is a primitive ring having nonzero socle with the field $\mathcal{C}$ as its associated division ring. By [21, p. 75] $\mathcal{Q}_{r}$ is isomorphic to a dense subring of the ring of linear transformations of a vector space $\mathcal{V}$ over $\mathcal{C}$, containing nonzero linear transformations of finite rank.

If $\operatorname{dim}_{\mathcal{C}} \mathcal{V}=k \geq 2$ is a finite positive integer, then $\mathcal{Q}_{r} \cong \mathcal{M}_{k}(\mathcal{C})$ and the conclusion follows from Lemma 2.11 .

Assume now that $\operatorname{dim}_{\mathcal{C}} \mathcal{V}=\infty$. As in Lemma 2 in [35], the set $\mathcal{S}=f(\mathcal{R})$ is dense on $\mathcal{R}$. By the fact that $\Phi\left(r_{1}, \ldots, r_{n}\right)=0$ is a generalized polynomial identity of $\mathcal{R}$, we know that $\mathcal{R}$ satisfies the generalized polynomial identity

$$
a x^{2}+q x^{2} q^{-1} b-\left(a x+q x q^{-1} b\right)^{2}
$$

and the required conclusions follows from Lemma 1.1.

\subsection{The proof of Proposition 2.1 in case of outer associated auto- morphism}

We finally prove Proposition 2.1 in the case $\alpha$ is an $X$-outer automorphism of $\mathcal{R}$. In light of Subsection 2.2, we consider $\alpha$ is not the identity map on $\mathcal{R}$.

In view of [13] we know that $\mathcal{R}$ and $\mathcal{Q}_{r}$ satisfy the same generalized polynomial identities with automorphisms. Therefore

$$
\begin{aligned}
\Phi\left(x_{1}, \ldots, x_{n}\right)= & a f\left(x_{1}, \ldots, x_{n}\right)^{2}+\alpha\left(f\left(x_{1}, \ldots, x_{n}\right)^{2}\right) b \\
& -\left(a f\left(x_{1}, \ldots, x_{n}\right)+\alpha\left(f\left(x_{1}, \ldots, x_{n}\right)\right) b\right)^{2}
\end{aligned}
$$

is also satisfied by $\mathcal{Q}_{r}$. Moreover, $\mathcal{Q}_{r}$ is a centrally closed prime $\mathcal{C}$-algebra. Note that if $b=0$ we are done by Subsection 2.2 .

We now suppose that $b \neq 0$. In this case, it follows from [14, Main Theorem] that $\Phi\left(x_{1}, \ldots, x_{n}\right)$ is a non-trivial generalized identity for $\mathcal{R}$ and for $\mathcal{Q}_{r}$. By $\left[22\right.$, Theorem 1] we get that $\mathcal{R C}$ has non-zero socle and $\mathcal{Q}_{r}$ is primitive. Since $\alpha$ is an outer automorphism and any $\left(x_{i}\right)^{\alpha}$-word degree in $\Phi\left(x_{1}, \ldots, x_{n}\right)$ is equal to 2 and $\operatorname{char}(\mathcal{R})=0$ or $\operatorname{char}(\mathcal{R})=p>2$, then by [14, Theorem 3], $\mathcal{Q}_{r}$ satisfies the generalized polynomial identity

$$
\begin{aligned}
\Phi\left(x_{1}, \ldots, x_{n}\right)= & a f\left(x_{1}, \ldots, x_{n}\right)^{2}+\left(f^{\alpha}\left(y_{1}, \ldots, y_{n}\right)^{2}\right) b \\
& -\left(a f\left(x_{1}, \ldots, x_{n}\right)+f^{\alpha}\left(y_{1}, \ldots, y_{n}\right) b\right)^{2},
\end{aligned}
$$


where we denote by $f^{\alpha}\left(x_{1}, \ldots, x_{n}\right)$ the polynomial obtained from $f\left(x_{1}, \ldots, x_{n}\right)$ by replacing each coefficient $\gamma_{\sigma}$ with $\alpha\left(\gamma_{\sigma}\right)$. Notice that $f^{\alpha}\left(x_{1}, \ldots, x_{n}\right)$ is not central valued on $\mathcal{R}$.

By $(2.11) \mathcal{Q}_{r}$ satisfies both

$$
a f\left(x_{1}, \ldots, x_{n}\right)^{2}-\left(a f\left(x_{1}, \ldots, x_{n}\right)\right)^{2}
$$

and

$$
\left(f^{\alpha}\left(y_{1}, \ldots, y_{n}\right)^{2}\right) b-\left(f^{\alpha}\left(y_{1}, \ldots, y_{n}\right) b\right)^{2}
$$

In light of Subsection 2.2 one has that:

- either $a=0$ or $a=1$;

- either $b=0$ or $b=1$.

Since $b \neq 0$, it follows that $b=1$ and $a=0$ or $a=1$. In case $a=0$, then $\mathcal{G}=\alpha$ and we are done.

Therefore, we suppose $a=1$ and prove that a contradiction follows. In fact, in this last case and by computations on (2.11), we have that $\mathcal{Q}_{r}$ satisfies

$$
f\left(x_{1}, \ldots, x_{n}\right) f^{\alpha}\left(y_{1}, \ldots, y_{n}\right) b+f^{\alpha}\left(y_{1}, \ldots, y_{n}\right) b f\left(x_{1}, \ldots, x_{n}\right) .
$$

By Lemma 2.13 it follows $f^{\alpha}\left(y_{1}, \ldots, y_{n}\right) b=0$ which implies the contradiction $b=0$.

\section{The proof of Theorem 1}

Let us first recall the following:

Fact 3.1. Let $\mathcal{R}$ be a prime ring, $\mathcal{D}$ be an $X$-outer skew derivation of $\mathcal{R}$ and $\alpha$ be an $X$-outer automorphism of $\mathcal{R}$. If $\Phi\left(x_{i}, \mathcal{D}\left(x_{i}\right), \alpha\left(x_{i}\right)\right)$ is a generalized polynomial identity for $\mathcal{R}$, then $\mathcal{R}$ also satisfies the generalized polynomial identity $\Phi\left(x_{i}, y_{i}, z_{i}\right)$, where $x_{i}, y_{i}$ and $z_{i}$ are distinct indeterminates ([16, Theorem 1]).

\subsection{The proof of Theorem 1}

As remarked in the Introduction, we can write $\mathcal{G}(x)=b x+d(x)$ for all $x \in \mathcal{R}, b \in \mathcal{Q}_{r}$ and $d$ is a skew derivation of $\mathcal{R}$ (see [8]). By [16, Theorem 2] we know that $\mathcal{R}$ and $\mathcal{Q}_{r}$ satisfy the same generalized polynomial identities with a single skew derivation. Thus $\mathcal{Q}_{r}$ satisfies

$$
\begin{aligned}
\Phi\left(x_{1}, \ldots, x_{n}, d\left(x_{1}\right), \ldots, d\left(x_{n}\right)\right)= & a f\left(x_{1}, \ldots, x_{n}\right)^{2}+d\left(f\left(x_{1}, \ldots, x_{n}\right)^{2}\right) \\
& -\left(a f\left(x_{1}, \ldots, x_{n}\right)+d\left(f\left(x_{1}, \ldots, x_{n}\right)\right)\right)^{2} .
\end{aligned}
$$

If $d$ is $X$-inner, then there exist $c \in \mathcal{Q}_{r}$ and $\alpha \in \operatorname{Aut}\left(\mathcal{Q}_{r}\right)$ such that $d(x)=$ $c x+\alpha(x) c$ for all $x \in \mathcal{R}$. In this case $\mathcal{G}(x)=(a+c) x+\alpha(x) c$ and by Proposition 2.1 either $\mathcal{G}(x)=x$ for all $x \in \mathcal{R}$, or $\mathcal{G}=\alpha$. 
Suppose that $d$ is $X$-outer and that $\alpha \in \operatorname{Aut}\left(\mathcal{Q}_{r}\right)$ is the associated automorphism of $d$. We denote by $f^{d}\left(x_{1}, \ldots, x_{n}\right)$ the polynomial obtained from $f\left(x_{1}, \ldots, x_{n}\right)$ by replacing each coefficient $\gamma_{\sigma}$ with $d\left(\gamma_{\sigma}\right)$. When $\alpha$ is the identity map on $\mathcal{R}$, then $d$ is an usual derivation of $\mathcal{R}$. And hence (3.1) implies that $\mathcal{R}$ satisfies

$$
\begin{aligned}
& a f\left(x_{1}, \ldots, x_{n}\right)^{2} \\
& +\left(f^{d}\left(x_{1}, \ldots, x_{n}\right)+\sum_{i} f\left(x_{1}, \ldots, d\left(x_{i}\right), \ldots, x_{n}\right)\right) f\left(x_{1}, \ldots, x_{n}\right) \\
& +f\left(x_{1}, \ldots, x_{n}\right)\left(f^{d}\left(x_{1}, \ldots, x_{n}\right)+\sum_{i} f\left(x_{1}, \ldots, d\left(x_{i}\right), \ldots, x_{n}\right)\right) \\
& -\left(a f\left(x_{1}, \ldots, x_{n}\right)+f^{d}\left(x_{1}, \ldots, x_{n}\right)+\sum_{i} f\left(x_{1}, \ldots, d\left(x_{i}\right), \ldots, x_{n}\right)\right)^{2} .
\end{aligned}
$$

In light of Kharchenko's Theorem in [23], by (3.2) it follows that $\mathcal{R}$ satisfies

$$
\begin{aligned}
& a f\left(x_{1}, \ldots, x_{n}\right)^{2} \\
& +\left(f^{d}\left(x_{1}, \ldots, x_{n}\right)+\sum_{i} f\left(x_{1}, \ldots, y_{i}, \ldots, x_{n}\right)\right) f\left(x_{1}, \ldots, x_{n}\right) \\
& +f\left(x_{1}, \ldots, x_{n}\right)\left(f^{d}\left(x_{1}, \ldots, x_{n}\right)+\sum_{i} f\left(x_{1}, \ldots, y_{i}, \ldots, x_{n}\right)\right) \\
& -\left(a f\left(x_{1}, \ldots, x_{n}\right)+f^{d}\left(x_{1}, \ldots, x_{n}\right)+\sum_{i} f\left(x_{1}, \ldots, y_{i}, \ldots, x_{n}\right)\right)^{2} .
\end{aligned}
$$

In particular

$$
\begin{aligned}
& \left(\sum_{i} f\left(x_{1}, \ldots, y_{i}, \ldots, x_{n}\right)\right) f\left(x_{1}, \ldots, x_{n}\right) \\
& +f\left(x_{1}, \ldots, x_{n}\right)\left(\sum_{i} f\left(x_{1}, \ldots, y_{i}, \ldots, x_{n}\right)\right) \\
& -\left(\sum_{i} f\left(x_{1}, \ldots, y_{i}, \ldots, x_{n}\right)\right)^{2}
\end{aligned}
$$

is a generalized identity for $\mathcal{R}$. Replacing any $y_{i}$ with $\left[u, x_{i}\right]$ for $u \in \mathcal{R}$ such that $u \notin \mathcal{Z}(\mathcal{R})$, one has that $\mathcal{R}$ satisfies the generalized polynomial identity

$$
\left[q, f\left(x_{1}, \ldots, x_{n}\right)^{2}\right]-\left[q, f\left(x_{1}, \ldots, x_{n}\right)\right]^{2}
$$

and by Subsection 2.2 we get the contradiction $u \in \mathcal{Z}(\mathcal{R})$.

Moreover if $d=0$, the required conclusion follows again from Subsection 2.2. Hence in what follows we always assume that $1_{\mathcal{R}} \neq \alpha \in \operatorname{Aut}(\mathcal{R})$ and $d \neq 0$. It 
should be remarked that

$$
\begin{aligned}
& d\left(\gamma_{\sigma} \cdot x_{\sigma(1)} \cdot x_{\sigma(2)} \cdots x_{\sigma(n)}\right) \\
= & d\left(\gamma_{\sigma}\right) x_{\sigma(1)} \cdot x_{\sigma(2)} \cdots x_{\sigma(n)}+\alpha\left(\gamma_{\sigma}\right) \sum_{j=0}^{n-1} \alpha\left(x_{\sigma(1)} \cdot x_{\sigma(2)} \cdots x_{\sigma(j)}\right) d\left(x_{\sigma(j+1)}\right) x_{\sigma(j+2)} \cdots x_{\sigma(n)} .
\end{aligned}
$$

So we have

$$
\begin{aligned}
& d\left(f\left(x_{1}, \ldots, x_{n}\right)\right) \\
= & f^{d}\left(x_{1}, \ldots, x_{n}\right)+\sum_{\sigma \in S_{n}} \alpha\left(\gamma_{\sigma}\right) \sum_{j=0}^{n-1} \alpha\left(x_{\sigma(1)} \cdot x_{\sigma(2)} \cdots x_{\sigma(j)}\right) d\left(x_{\sigma(j+1)}\right) x_{\sigma(j+2)} \cdots x_{\sigma(n)} .
\end{aligned}
$$

Since $\mathcal{Q}_{r}$ satisfies $\Phi\left(x_{1}, \ldots, x_{n}, d\left(x_{1}\right), \ldots, d\left(x_{n}\right)\right)$, then it also satisfies

$$
\begin{aligned}
& a f\left(x_{1}, \ldots, x_{n}\right)^{2} \\
& +\left(f^{d}\left(x_{1}, \ldots, x_{n}\right)+\sum_{\sigma \in S_{n}} \alpha\left(\gamma_{\sigma}\right) \sum_{j=0}^{n-1} \alpha\left(x_{\sigma(1)} \cdot x_{\sigma(2)} \cdots x_{\sigma(j)}\right) d\left(x_{\sigma(j+1)}\right) x_{\sigma(j+2)} \cdots x_{\sigma(n)}\right) f\left(x_{1}, \ldots, x_{n}\right) \\
& +f^{\alpha}\left(\alpha\left(x_{1}\right), \ldots, \alpha\left(x_{n}\right)\right)\left(f^{d}\left(x_{1}, \ldots, x_{n}\right)+\sum_{\sigma \in S_{n}} \alpha\left(\gamma_{\sigma}\right) \sum_{j=0}^{n-1} \alpha\left(x_{\sigma(1)} \cdot x_{\sigma(2)} \cdots x_{\sigma(j)}\right) d\left(x_{\sigma(j+1)}\right) x_{\sigma(j+2)} \cdots x_{\sigma(n)}\right) \\
& -\left(a f\left(x_{1}, \ldots, x_{n}\right)+f^{d}\left(x_{1}, \ldots, x_{n}\right)+\sum_{\sigma \in S_{n}} \alpha\left(\gamma_{\sigma}\right) \sum_{j=0}^{n-1} \alpha\left(x_{\sigma(1)} \cdot x_{\sigma(2)} \cdots x_{\sigma(j)}\right) d\left(x_{\sigma(j+1)}\right) x_{\sigma(j+2)} \cdots x_{\sigma(n)}\right)^{2} .
\end{aligned}
$$

By $\left[16\right.$, Theorem 1] it follows that $\mathcal{Q}_{r}$ satisfies $\Phi\left(x_{1}, \ldots, x_{n}, y_{1}, \ldots, y_{n}\right)$, that is

$$
\begin{aligned}
& a f\left(x_{1}, \ldots, x_{n}\right)^{2} \\
& +\left(f^{d}\left(x_{1}, \ldots, x_{n}\right)+\sum_{\sigma \in S_{n}} \alpha\left(\gamma_{\sigma}\right) \sum_{j=0}^{n-1} \alpha\left(x_{\sigma(1)} \cdot x_{\sigma(2)} \cdots x_{\sigma(j)}\right) y_{\sigma(j+1)} x_{\sigma(j+2)} \cdots x_{\sigma(n)}\right) f\left(x_{1}, \ldots, x_{n}\right) \\
& +f^{\alpha}\left(\alpha\left(x_{1}\right), \ldots, \alpha\left(x_{n}\right)\right)\left(f^{d}\left(x_{1}, \ldots, x_{n}\right)+\sum_{\sigma \in S_{n}} \alpha\left(\gamma_{\sigma}\right) \sum_{j=0}^{n-1} \alpha\left(x_{\sigma(1)} \cdot x_{\sigma(2)} \cdots x_{\sigma(j)}\right) y_{\sigma(j+1)} x_{\sigma(j+2)} \cdots x_{\sigma(n)}\right) \\
& -\left(a f\left(x_{1}, \ldots, x_{n}\right)+f^{d}\left(x_{1}, \ldots, x_{n}\right)+\sum_{\sigma \in S_{n}} \alpha\left(\gamma_{\sigma}\right) \sum_{j=0}^{n-1} \alpha\left(x_{\sigma(1)} \cdot x_{\sigma(2)} \cdots x_{\sigma(j)}\right) y_{\sigma(j+1)} x_{\sigma(j+2)} \cdots x_{\sigma(n)}\right)^{2} .
\end{aligned}
$$

In particular, $\mathcal{Q}_{r}$ satisfies

$$
\begin{aligned}
& a f\left(x_{1}, \ldots, x_{n}\right)^{2} \\
& +\left(\sum_{\sigma \in S_{n}} \alpha\left(\gamma_{\sigma}\right) \sum_{j=0}^{n-1} \alpha\left(x_{\sigma(1)} \cdot x_{\sigma(2)} \cdots x_{\sigma(j)}\right) y_{\sigma(j+1)} x_{\sigma(j+2)} \cdots x_{\sigma(n)}\right) f\left(x_{1}, \ldots, x_{n}\right) \\
& +f^{\alpha}\left(\alpha\left(x_{1}\right), \ldots, \alpha\left(x_{n}\right)\right)\left(\sum_{\sigma \in S_{n}} \alpha\left(\gamma_{\sigma}\right) \sum_{j=0}^{n-1} \alpha\left(x_{\sigma(1)} \cdot x_{\sigma(2)} \cdots x_{\sigma(j)}\right) y_{\sigma(j+1)} x_{\sigma(j+2)} \cdots x_{\sigma(n)}\right)
\end{aligned}
$$




$$
-\left(\sum_{\sigma \in S_{n}} \alpha\left(\gamma_{\sigma}\right) \sum_{j=0}^{n-1} \alpha\left(x_{\sigma(1)} \cdot x_{\sigma(2)} \cdots x_{\sigma(j)}\right) y_{\sigma(j+1)} x_{\sigma(j+2)} \cdots x_{\sigma(n)}\right)^{2} .
$$

Here we divide the argument in two subcases. Let us first consider the case when $\alpha$ is an inner automorphism of $\mathcal{R}$. Then there exists an invertible element $q \in \mathcal{Q}_{r}$ such that $\alpha(x)=q x q^{-1}$ for all $x \in \mathcal{R}$. Since $1_{\mathcal{R}} \neq \alpha \in \operatorname{Aut}(\mathcal{R})$, we may assume that $q \notin \mathcal{C}$. Moreover, it is clear that $\alpha\left(\gamma_{\sigma}\right)=\gamma_{\sigma}$ for all coefficients involved in $f\left(x_{1}, \ldots, x_{n}\right)$.

If we choose in (3.8) $x_{1}=0$ and $y_{1}=q z_{1}$, we have that

$$
\left(q f\left(z_{1}, x_{2}, \ldots, x_{n}\right)\right)^{2}
$$

is satisfied by $\mathcal{Q}_{r}$. Therefore by the result in [33] and since $f\left(x_{1}, \ldots, x_{n}\right)$ is not an identity for $\mathcal{Q}_{r}$, we get the contradiction $q=0$.

We now assume that $\alpha$ is $X$-outer. In light of Fact 3.1 and the relation (3.8), $\mathcal{Q}_{r}$ satisfies the generalized polynomial identity

$$
\begin{aligned}
& \text { af }\left(x_{1}, \ldots, x_{n}\right)^{2}+\left(\sum_{\sigma \in S_{n}} \alpha\left(\gamma_{\sigma}\right) \sum_{j=0}^{n-1} z_{\sigma(1)} \cdots z_{\sigma(j)} y_{\sigma(j+1)} x_{\sigma(j+2)} \cdots x_{\sigma(n)}\right) f\left(x_{1}, \ldots, x_{n}\right) \\
& +f^{\alpha}\left(\alpha\left(x_{1}\right), \ldots, \alpha\left(x_{n}\right)\right)\left(\sum_{\sigma \in S_{n}} \alpha\left(\gamma_{\sigma}\right) \sum_{j=0}^{n-1} z_{\sigma(1)} \cdots z_{\sigma(j)} y_{\sigma(j+1)} x_{\sigma(j+2)} \cdots x_{\sigma(n)}\right) \\
& -\left(\sum_{\sigma \in S_{n}} \alpha\left(\gamma_{\sigma}\right) \sum_{j=0}^{n-1} z_{\sigma(1)} \cdots z_{\sigma(j)} y_{\sigma(j+1)} x_{\sigma(j+2)} \cdots x_{\sigma(n)}\right)^{2} .
\end{aligned}
$$

Let us write

$$
\sum_{\sigma \in S_{n-1}} \alpha\left(\gamma_{\sigma}\right) x_{\sigma(1)} \cdots x_{\sigma(i-1)} x_{\sigma(i+1)} \cdots x_{\sigma(n)}=t_{i}\left(x_{1}, \ldots, x_{i-1}, x_{i+1}, \ldots, x_{n}\right),
$$

where any $t_{j}$ is a multilinear polynomial of degree $n-1$ and $x_{j}$ never appears in any monomial of $t_{j}$.

Thus $f^{\alpha}\left(x_{1}, \ldots, x_{n}\right)=\sum_{i} x_{i} t_{i}\left(x_{1}, \ldots, x_{i-1}, x_{i+1}, \ldots, x_{n}\right)$.

Moreover, since $f^{\alpha}\left(x_{1}, \ldots, x_{n}\right) \neq 0$, then there exists $i \geq 1$ such that

$$
t_{i}\left(x_{1}, \ldots, x_{i-1}, x_{i+1}, \ldots, x_{n}\right) \neq 0 .
$$

For all $k=1, \ldots, n$, we choose in $(3.9)$ :

- $x_{k}=0$;

- for all $j \neq k, y_{\sigma(j)}=0$;

- for all $j \neq k, z_{\sigma(j)}=0$.

Therefore by (3.9) we assert that $\mathcal{Q}_{r}$ satisfies the generalized polynomial identity

$$
\left(y_{k} t_{k}\left(x_{1}, \ldots, x_{k-1}, x_{k+1}, \ldots, x_{n}\right)\right)^{2} .
$$

As above, by [33] we have that any polynomial $t_{k}\left(x_{1}, \ldots, x_{k-1}, x_{k+1}, \ldots, x_{n}\right)$ is an identity for $\mathcal{Q}_{r}$ and this leads to the contradiction $f^{\alpha}\left(x_{1}, \ldots, x_{n}\right)=0$. 


\section{References}

[1] E. Albaş and N. Argaç, Generalized derivations of prime rings, Algebra Colloq. 11 (2004), no. 3, 399-410.

2] S. Ali and S. Huang, On generalized Jordan $(\alpha, \beta)$-derivations that act as homomorphisms or anti-homomorphisms, J. Algebra Computat. Appl. 1 (2011), no. 1, 13-19.

[3] A. Ali and D. Kumar, Derivation which acts as a homomorphism or as an antihomomorphism in a prime ring, Int. Math. Forum 2 (2007), no. 21-24, 1105-1110.

[4] —, Generalized derivations as homomorphisms or as anti-homomorphisms in a prime ring, Hacet. J. Math. Stat. 38 (2009), no. 1, 17-20.

5] A. Asma, N. Rehman, and A. Shakir, On Lie ideals with derivations as homomorphisms and anti-homomorphisms, Acta Math. Hungar. 101 (2003), no. 1-2, 79-82.

[6] K. I. Beidar, W. S. Martindale III, and A. V. Mikhalev, Rings with Generalized Identities, Pure and Applied Math., Dekker, New York, 1996.

[7] H. E. Bell and L. C. Kappe, Rings in which derivations satisfy certain algebraic conditions, Acta Math. Hungar. 53 (1989), no. 3-4, 339-346.

[8] J. -C. Chang, On the identity $h(x)=a f(x)+g(x) b$, Taiwanese J. Math. 7 (2003), no. $1,103-113$.

9] _ Generalized skew derivations with annihilating Engel conditions, Taiwanese J. Math. 12 (2008), no. 7, 1641-1650.

[10] _ Generalized skew derivations with nilpotent values on Lie ideals, Monatsh. Math. 161 (2010), no. 2, 155-160.

[11] H.-W. Cheng and F. Wei, Generalized skew derivations of rings, Adv. Math. (China) 35 (2006), no. 2, 237-243.

[12] C. -L. Chuang, GPIs having coefficients in Utumi quotient rings, Proc. Amer. Math. Soc. 103 (1988), no. 3, 723-728.

[13] - Differential identities with automorphisms and antiautomorphisms I, J. Algebra 149 (1992), no. 2, 371-404.

[14] , Differential identities with automorphisms and antiautomorphisms II, J. Algebra 160 (1993), no. 1, 130-171.

[15] C. -L. Chuang and T.-K. Lee, Rings with annihilator conditions on multilinear polynomials, Chinese J. Math. 24 (1996), no. 2, 177-185.

[16] _ Identities with a single skew derivation, J. Algebra 288 (2005), no. 1, 59-77.

[17] V. De Filippis, Generalized derivations as Jordan homomorphisms on Lie ideals and right ideals, Acta Math. Sin. 25 (2009), no. 12, 1965-1974.

[18] _ A products of two generalized derivations on polynomials in prime rings, Collect. Math. 61 (2010), no. 3, 303-322.

[19] _ Annihilators of power values of generalized derivations on multilinear polynomials, Bull. Aust. Math. Soc. 80 (2009), no. 2, 217-232.

[20] I. N. Herstein, Topics in Ring Theory, Univ. of Chicago Press, Chicago 1969.

[21] N. Jacobson, Structure of Rings, Amer. Math. Soc., Providence, RI, 1964

[22] V. K. Kahrchenko, Generalized identities with automorphisms, Algebra and Logic 14 (1975), 132-148.

[23] Differential identities of prime rings, Algebra Log. 17 (1978), 155-168.

[24] T.-K. Lee, Derivations with invertible values on a multilinear polynomial, Proc. Amer. Math. Soc. 119 (1993), no. 4, 1077-1083.

[25] — Generalized skew derivations characterized by acting on zero products, Pacific J. Math. 216 (2004), no. 2, 293-301.

[26] U. Leron, Nil and power central polynomials in rings, Trans. Amer. Math. Soc. 202 (1975), 97-103.

[27] K.-S Liu, Differential identities and constants of algebraic automorphisms in prime rings, Ph.D. Thesis, National Taiwan University, 2006. 
[28] S.-J. Luo, Posner's theorems with skew derivations, Master Thesis, National Taiwan University, 2007.

[29] W. S. Martindale III, Prime rings satisfying a generalized polynomial identity, J. Algebra 12 (1969), 576-584.

[30] L. Oukhtite, S. Salhi, and L. Taoufiq, $\sigma$-Lie ideals with derivations as homomorphisms and anti-homomorphisms, Int. J. Algebra 1 (2007), no. 5-8, 235-239.

[31] N. Rehman, On generalized derivations as homomorphisms and anti-homomorphisms, Glas. Mat. III ser.39 N.1 (2004), 27-30.

[32] G. Scudo, Generalized derivations acting as Lie on polynomials in prime rings, Southeast Asian Bull. Math. 38 (2014), 563-572.

[33] Y. Wang, Generalized derivations with power-central values on multilinear polynomials, Algebra Colloq. 13 (2006), no. 3, 405-410.

[34] Y. Wang and H. You, Derivations as homomorphisms or anti-homomorphisms on Lie ideals, Acta Math. Sin. 23 (2007), no. 6, 1149-1152.

[35] T.-L. Wong, Derivations with power central values on multilinear polynomials, Algebra Colloq. 3 (1996), no. 4, 369-378.

[36] X. Xu, J. Ma, and F. Niu, Compositions, derivations and polynomials, Indian J. Pure Appl. Math. 44 (2013), no. 4, 543-556.

Department of Mathematics and Computer Science UNIVERSITY OF MESSINA

Viale S. D'Alcontres 31, 98166, Messina, Italy

E-mail address: defilippis@unime.it 